\title{
RESEARCH-IN-BRIEF
}

\section{Mit zweierlei Maß gemessen? Ein Vergleich der für Politiker und Fußballer bei Steuer- und Drogendelikten geforderten Konsequenzen}

\begin{abstract}
Not as bad as it seems? A comparison of the consequences demanded for soccer players and politicians who have committed a tax offence or a drug-related offence
\end{abstract}

\author{
Catharina Vögele
}


Catharina Vögele (Dr.), Institut für Kommunikationswissenschaft, Universität Hohenheim, Fruwirthstraße 46, 70599 Stuttgart, Germany; Contact: c.voegele(at)uni-hohenheim.de. 


\title{
Mit zweierlei Maß gemessen? Ein Vergleich der für Politiker und Fußballer bei Steuer- und Drogendelikten geforderten Konsequenzen
}

\author{
Not as bad as it seems? A comparison of the consequences \\ demanded for soccer players and politicians who have committed \\ a tax offence or a drug-related offence
}

\section{Catharina Vögele}

\begin{abstract}
Zusammenfassung: Die Studie analysiert, welche Konsequenzen für Fußballer im Vergleich zu Politiker*innen bei einem Steuer- sowie einem Drogendelikt gefordert werden und ob sich dabei Unterschiede zeigen, die auf die unterschiedliche Relevanz dieser Vergehen für den jeweiligen Beruf und auf die Verwendung unterschiedlicher moralischer Begründungsstrategien zurückzuführen sind. Diese Begründungsstrategien können Menschen einsetzen, um das unmoralische Verhalten von Personen des öffentlichen Lebens, die sie schätzen, zu erklären und damit ein positives Bild dieser beizubehalten. Hierzu können sie entweder das moralische Fehlverhalten der Person herunterspielen (Moral Rationalization) oder die Leistungsbewertung der Person von der Bewertung ihrer Moral abspalten (Moral Decoupling). Mithilfe einer Online-Befragung im Experimentaldesign (2x2-Between-Subjects-Design), bei der die Probanden*innen $(N=447)$ einen Artikel zu einem fiktiven österreichischen Politiker oder Fußballer lasen, der entweder ein Steuer- oder ein Drogendelikt begangen hat, analysieren wir unsere Fragestellung. Das Steuerdelikt war das rollennahe, berufsrelevante Fehlverhalten des Politikers, das Drogendelikt das rollennahe Vergehen des Fußballers. Im Anschluss an den Artikel fragen wir die Probanden*innen, wie stark sie bestimmte Konsequenzen für den Politiker bzw. Fußballer fordern. Die Ergebnisse zeigen, dass bei rollennahem moralischem Fehlverhalten sowohl für den Politiker als auch für den Fußballer im Vergleich zu rollenfernem Fehlverhalten stärkere Konsequenzen gefordert werden. Ein Teil dieses Effekts kann mithilfe des Einsatzes der Moral Decoupling Strategie erklärt werden. So führt ein rollennahes Fehlverhalten dazu, dass die Rezipient*innen die Moral Decoupling Strategie weniger intensiv einsetzen und deshalb den Konsequenzen stärker zustimmen. Für die Moral Rationalization Strategie zeigt sich dieser indirekte Effekt jedoch nicht.
\end{abstract}

Schlagwörter: Online-Experiment, Moral Decoupling, Steuer- und Drogendelikt, Sportkommunikation, politische Kommunikation.

Abstract: This study analyzes which consequences are demanded for a soccer player who has committed a tax offence or a drug-related offence in comparison to a politician and whether differences can be identified depending on the relevance of the offence for the job and the use of different moral reasoning strategies. These strategies help persons to maintain support for public figures who acted immorally. They can use the moral decoupling strategy by sepa- 
rating the judgment of the public figure's performance from the judgment of its morality or they can use the moral rationalization strategy by playing down the immoral action. We conducted a $2 \times 2$ between-subjects online-experiment $(N=447)$. The participants read a newspaper article about either a soccer player or a politician committing a tax offence or a drug-related offence. We defined the tax offence as relevant for the job of the politician and the drug-related offence as relevant for the soccer player. After reading the article the participants had to answer how strongly they demand different consequences for the politician respectively soccer player. Our results show that the consequences were demanded more strongly when the offence was relevant for the job. This effect can be explained by the more intensive use of the moral decoupling strategy. The participants less intensively used moral decoupling when the offence was job-relevant and thus demanded harder consequences. We couldn't identify this indirect effect concerning moral rationalization.

Keywords: Online-experiment, moral decoupling, tax and drug-related offence, sports communication, political communication.

\section{Einleitung}

Seit Dezember 2016 sind aufgrund der „Football Leaks“-Veröffentlichungen des Spiegels und seiner Medienpartner Steuerdelikte prominenter Fußballer und Trainer wie Cristiano Ronaldo, Xabi Alonso oder José Mourinho bekannt geworden (Buschmann \& Wulzinger, 2017). Juristische Konsequenzen hatten diese Enthüllungen in der Form, dass die Staatsanwaltschaft in Madrid Strafanzeigen wegen Steuerhinterziehung gestellt hat (Fleischhauer, 2018; "Staatsanwaltschaft zeigt Cristiano", 2017; „Verdacht auf Steuerhinterziehung“, 2017). In den Medien wurde über diese Delikte zwar berichtet, auf einen Aufschrei auf Seiten der Öffentlichkeit ließen diese Veröffentlichungen jedoch warten. Es entstand vielmehr der Eindruck: „Der Fußball verzeiht viel, er ist ein eigener Kosmos“ (Hummel, 2017). In der Unterhaltungsmaschinerie Fußball scheint die Frage nach der Integrität und der Moral dieses Verhaltens, das nicht direkt mit den Leistungen der Fußballer auf dem Platz zu tun hat, in den Hintergrund zu rücken. Worauf lässt sich das zurückführen? Ist es wirklich so, dass das Verhalten von Fußballern, das nicht direkt mit ihrer sportlichen Leistung in Verbindung steht, für deren Bewertung und Beliebtheit in der Öffentlichkeit keine Rolle zu spielen scheint? Und wenn ja, wie lässt sich das erklären?

Um diese Fragen zu beantworten, zogen wir als Vergleichsobjekt Politiker*innen heran. Können diese, wenn sie sich Steuerdelikte zu Schulden kommen lassen, ebenfalls auf ein mildes Urteil in der Bevölkerung hoffen? Da Politiker*innen unter anderem für die Steuergesetzgebung verantwortlich sind, stellt ein dementsprechendes Vergehen ein Fehlverhalten dar, das stärker mit ihrem Beruf verknüpft ist. Deshalb vermuteten wir, dass für Politiker*innen bei Steuervergehen härtere Konsequenzen gefordert werden im Vergleich zu Fußballern. Diese Vermutung legen auch die sogenannten Moral Reasoning Strategien nahe, die die theoretische Grundlage unserer Studie bilden und erklären, wie Menschen das unmoralische 
Verhalten von sich selbst oder von Personen des öffentlichen Lebens erklären (Symmank \& Hoffmann, 2017, S. 962). $\mathrm{Da}$ die Bevölkerung über das moralische Fehlverhalten von bekannten Persönlichkeiten fast ausschließlich aus den Medien erfährt, steht in dieser Studie die Frage im Mittelpunkt, ob Medienrezipient"innen Steuerdelikte bei Fußballern im Vergleich zu Politiker"innen weniger gravierend einschätzen und daher Konsequenzen für diese weniger intensiv fordern, da diese Art des Fehlverhalten für den Beruf des Fußballers weniger relevant erscheint als für den Politiker oder die Politikerin. Um zu analysieren, ob die Relevanz des Fehlverhaltens für den jeweiligen Beruf für die Beurteilung eines unmoralischen Verhaltens entscheidend ist, zogen wir für unsere Analyse zusätzlich ein Drogendelikt heran. Bei der Einnahme leistungssteigernder Drogen handelt es sich um ein Fehlverhalten, das für Fußballer, deren Leistung stark von ihrer körperlichen Verfassung abhängig ist, berufsrelevant ist, für Politiker"innen jedoch in wesentlich geringerem Ausmaß. Die Beurteilung der Konsequenzen für Politiker*innen und Fußballer bei Steuer- und Drogenvergehen verglichen wir mithilfe einer Online-Befragung im Experimentaldesign. Dabei bekamen die Versuchspersonen entweder einen Zeitungsartikel zu lesen, in dem a) über ein Steuervergehen eines Politikers oder b) eines Fußballers berichtet wurde oder c) über ein Drogendelikt eines Politikers oder d) eines Fußballers. Im Mittelpunkt standen die Fragen, welche Konsequenzen Rezipient*innen für Fußballer im Vergleich zu Politikern bei einem Steuer- sowie einem Drogendelikt fordern und ob sich dabei Unterschiede zeigen, die sich durch die Verwendung unterschiedlicher moralischer Begründungsstrategien erklären lassen.

\section{Theoretische Grundlage und Forschungsstand}

Der Sport und insbesondere der ProfiFußball sowie auch die Politik sind in der heutigen Mediengesellschaft in großem Maße medienvermittelt. Die Bürger*innen erlangen Informationen zu diesen gesellschaftlichen Teilsystemen insbesondere und in erster Linie über die Massenmedien (vgl. z. B. Gleich, 2001, S. 167; Jarren \& Donges, 2011, S. 23; Schulz, 2011, S. 23). Sowohl der Sport wie auch die Politik haben sich deshalb an die Logik der Massenmedien angepasst, sich medialisiert, um besonders präsent in der Berichterstattung zu sein (vgl. z. B. Heinecke, 2014; Jarren \& Donges, 2011; Meyen, 2014; Schulz, 2011). Fällt ein Fußballer oder ein Politiker bzw. eine Politikerin durch ein Fehlverhalten auf, ist es am wahrscheinlichsten, dass die Bürger*innen von diesem Vergehen über die Medienberichterstattung erfahren. Denn gerade bezüglich des Aufdeckens von Missständen in der Gesellschaft kommt den Massenmedien eine zentrale Rolle zu. So sind es häufig diese, die erst dafür sorgen, dass ein Missstand oder ein Vergehen öffentlich wird (Schulz, 2011, S. 102). Diese Art von Medienpräsenz streben Personen des öffentlichen Lebens natürlich nicht an, da sie für sie von Nachteil ist. Sie wollen sich in so einem Fall deshalb eher von den Medien distanzieren. Diese Abschirmstrategie, die Birkner und Nölleke (2016, S. 380) bei männlichen Fußballprofis aufgrund der Omnipräsenz der Medien im Fußball identifizieren konnten, ist 
neben der Anpassung an die Logik der Massenmedien, um dortige Präsenz zu erzielen, eine weitere Strategie für den Umgang prominenter Akteure mit Medien.

In den Medien setzt im Anschluss an die Berichterstattung über Vergehen von Personen des öffentlichen Lebens häufig eine Diskussion und damit ein publizistischer Konflikt darüber ein, wie das Fehlverhalten moralisch zu bewerten ist und vor allem welche Konsequenzen es nach sich ziehen sollte (Kepplinger, 2009, S. 9). Von solchen moralischen Debatten wird die Berichterstattung über Politik- sowie über Sportskandale geprägt (Böcking, 2007, S. 505; Kepplinger, 2009, S. 7). Die medialen Debatten über die Frage, welche Folgen ein Fehlverhalten für eine öffentliche Person haben sollte, werden von den Medienrezipient*innen verfolgt. Auch sie setzen sich folglich mit dieser Frage auseinander und werden dabei in ihrer Einschätzung unter anderem von der Debatte in der Medienöffentlichkeit geprägt. Auf diesen Einschätzungen der Rezipient"innen liegt der Fokus unserer Studie. Im Anschluss an die Veröffentlichung der Steuerdelikte im Rahmen der Football Leaks war zu beobachten, dass das Fußballpublikum die Delikte zum Großteil ,, achselzuckend zur Kenntnis" (Hummel, 2017, S. 2) nahm und keine strengen Konsequenzen für die Beschuldigten forderte. Bei der Beantwortung der Frage, worauf diese Gleichgültigkeit zurückzuführen sein könnte, helfen uns sogenannte Moral Reasoning Strategien weiter.

Menschen können auf diese Moral Reasoning Strategien zurückgreifen, wenn sie ihr eigenes unmoralisches Verhalten erklären wollen, aber auch wenn es um die Erklärung des Fehlver- haltens einer öffentlichen (Medien)Person geht, die den Rezipient*innen bekannt ist (Symmank \& Hoffmann, 2017, S. 962-963). Vor allem, wenn die Rezipient*innen dieser bekannten Persönlichkeit gegenüber positiv eingestellt sind. Dann befinden sie sich nämlich in einem Dilemma. Sie möchten das positive Bild der Person beibehalten, gleichzeitig aber auch die eigenen moralischen Standards nicht über Bord werfen (Bhattacharjee, Berman, \& Reed, 2013, S. 1168). Das Fehlverhalten des Protagonisten, von dem die Rezipient*innen in den Medien erfahren, führt dazu, dass deren positive Voreinstellungen zu ihm und das Wissen über das Fehlverhalten in einer dissonanten Beziehung zueinander stehen. Nach der Theorie kognitiver Dissonanz von Festinger (1957, S. 3) neigt der Mensch dazu, eine solche Dissonanz zu reduzieren, weil sie ihm unangenehm ist. Hierbei kann der Mensch auf zwei unterschiedliche Moral Reasoning Strategien zurückgreifen: die Moral Rationalization Strategie oder die Moral Decoupling Strategie.

Bei der Rationalisierungsstrategie, dem Moral Rationalization, wird das unmoralische Verhalten der bekannten Persönlichkeit mithilfe unterschiedlicher Begründungen heruntergespielt und verharmlost (Bhattacharjee et al., 2013, S. 1168). Der bekannteste und elaborierteste theoretische Ansatz hierbei ist das Moral Disengagement, das auf Bandura (1991, 1999) zurückgeht. Bandura unterscheidet insgesamt vier Strategien, die angewandt werden können, um unmoralische Verhaltensweisen zu rechtfertigen (Bandura, 1991, 1999; Bandura et al., 1996). Erstens kann das unmoralische Verhalten so umgedeutet werden, dass es nicht mehr als moralisch verwerflich betrachtet 
wird. In unserem Beispiel könnte die Finanzierung einer Gesellschaft über Steuern beispielsweise insgesamt in Frage gestellt werden und damit ein Steuervergehen auch nicht mehr als unmoralisch betrachtet werden. Zweitens kann die Rolle des Schuldigen beim Fehlverhalten verharmlost werden. Im Beispiel des Steuervergehens könnte die Verantwortung hierfür dem Steuerberater des Politikers bzw. Fußballers zugeschrieben werden oder beim Drogenmissbrauch dem Arzt. Drittens kann der Schaden, der durch die Aktion entstanden ist, relativiert und kleingeredet werden. So kann erwähnt werden, dass ein Steuervergehen und ein Drogenvergehen im Vergleich $\mathrm{zu}$ anderem Fehlverhalten nicht so schwerwiegend sind, da hierbei keine anderen Menschen körperlich zu Schaden kommen. Schließlich kann das Opfer des Fehlverhaltens beschuldigt und entmenschlicht werden, was jedoch bei unseren Beispielen nur schwer vorstellbar ist (Bandura, 1991, 1999; Bandura et al., 1996; Bhattacharjee et al., 2013, S. 1168). Nachteil all dieser Begründungen ist, dass bei dieser Form der Relativierung das unmoralische Verhalten trotz allem geduldet werden muss (Bhattacharjee et al., 2013, S. 1169). Die eigenen moralischen Standards müssen also ein stückweit fallengelassen werden.

Mit dem Moral Decoupling bietet sich jedoch eine zweite Strategie an, die den Vorteil hat, dass hier die eigenen moralischen Standards bei der Beurteilung nicht in Frage gestellt werden müssen. Diese Strategie ist deshalb für die Rezipient*innen einfacher anzuwenden und auch leichter zu rechtfertigen (Bhattacharjee et al., 2013, S. 1168-1169). Das Moral Decoupling ist ein psychologischer Prozess, bei dem der Beurteilende die Bewertung der Leistung des Bewerteten in seinem Job von der Bewertung seiner Moral abspaltet. Durch diese Trennung der moralischen Bewertung von der Leistungsbewertung müssen die Rezipient"innen ihre moralischen Standards nicht anpassen (ebd.). Die Moralbewertung wird hierbei der Leistungsbewertung untergeordnet. Wenn das Fehlverhalten folglich nicht direkt in Bezug zu dem Beruf der entsprechenden Person steht, wird es auch nicht bei der Beurteilung ihrer Leistung in diesem Bereich beachtet (Bhattacharjee et al., 2013, S. 1169). Diese Trennung der Leistungsbewertung von der moralischen Bewertung kann aber natürlich nur bei solchem Fehlverhalten sinnvoll vorgenommen werden, das sich nicht auf den Beruf der Person bezieht. In unserem Beispiel scheint das Moral Decoupling deshalb vor allem bei Fußballern und Steuerdelikten sowie bei Politiker*innen und Drogenvergehen als Rechtfertigungsstrategie zweckmäßig zu sein, da hier das Fehlverhalten jeweils rollenfern, also nicht im jeweiligen Berufsfeld anzusiedeln ist. Die Frage, ob Rezipient*innen auf die Moral Reasoning Strategien zurückgreifen, um das moralische Fehlverhalten einer Person des öffentlichen Lebens zu rechtfertigen, hängt also stark davon $a b$, ob es sich um ein rollenfernes Fehlverhalten handelt, das keinen Bezug zum Beruf der Person hat, oder um ein rollennahes Fehlverhalten, bei dem ein enger $\mathrm{Zu}-$ sammenhang zum Beruf besteht. Wir fokussieren dabei in unserem Experiment nicht die Rechtfertigung des Fehlverhaltens, sondern die Konsequenzen, die von den Rezipienten für den Schuldigen gefordert werden. Dies tun wir, da öffentliche Debatten zu eindeutig negativem Fehlverhalten sehr stark da- 
von geprägt sind, welche Konsequenzen für die Beschuldigten gefordert werden (Kepplinger, 2009, S. 9). Wir gehen davon aus, dass die geforderten Konsequenzen eine der Rechtfertigung nachgelagerte Einschätzung sind und dass das Fordern von Konsequenzen somit ein guter Indikator für die Reaktion auf ein Fehlverhalten in der Öffentlichkeit ist. Die Nutzung von Moral Reasoning Strategien müsste deshalb auch die Zustimmung zu Konsequenzen beeinflussen.

Wie wir im Methodenteil berichten werden, greifen wir in unserem Experimentaldesign auf Zeitungsartikel als Stimuli zurück, die einen fiktiven Politiker bzw. Fußballer mit seinen Erfolgen vorstellen und anschließend über ein Fehlverhalten dieses Politikers bzw. Fußballers berichten. $\mathrm{Zu}$ diesen fiktiven Politikern bzw. Fußballern können die Rezipient*innen natürlich keine positiven Voreinstellungen haben, die sie dazu motivieren würde, Moral Reasoning Strategien einzusetzen. Wir gehen aber trotzdem davon aus, dass Moral Reasoning Strategien von den Rezipient*innen auch in diesen Fällen genutzt werden. Entscheidend hierfür ist dann nicht mehr die positive Grundeinstellung zu einem speziellen Politiker oder einer Politikerin bzw. einem speziellen Fußballer, sondern zum Beruf des Politikers oder der Politikerin bzw. des Fußballers im Allgemeinen. Die Voreinstellung zur spezifischen öffentlichen Person rückt folglich in den Hintergrund, die Voreinstellung zum Berufsfeld des Politikers oder der Politikerin bzw. des Fußballers wird wichtiger und kann erklären, warum die Rezipient*innen auf Moral Reasoning Strategien zurückgreifen.

Hierbei spielen die Unterschiede der zwei gesellschaftlichen Teilbereiche der
Politik und des Sports eine wichtige Rolle. Währenddessen der Sport und im Speziellen der Mediensport insbesondere zur Unterhaltung und Rekreation der Bevölkerung beitragen, ist die Politik vor allem dazu da, das Zusammenleben in der Gesellschaft auf Grundlage kollektiv verbindlicher Entscheidungen zu regeln und zu gestalten (vgl. z. B. Easton, 1953, S. 129-134; Nölleke \& Blöbaum, 2012, S. 167). Die Rezeption von Politik- und Sportberichterstattung geht deshalb auch auf unterschiedliche Motive zurück. Der Mediensport wird insbesondere zu Unterhaltungszwecken und zur Flucht aus dem Alltag und damit aus primär affektiven und emotionalen Gründen genutzt (vgl. z. B. Nölleke \& Blöbaum, 2012, S. 175; Raney, 2008, S. 70; Stiehler, 2007, S. 182). Bei der Rezeption von Politikberichterstattung stehen dagegen stärker kognitive Informationsbedürfnisse im Vordergrund, da die Medien in einer funktionierenden Demokratie die Aufgabe der Information sowie Kritik haben, um den Bürger*innen eine politische Meinungsbildung zu ermöglichen (Schulz, 2011, S. 54). Da der Sport im Vergleich zur Politik also einen stärker unterhaltenden Charakter hat, ist davon auszugehen, dass die Bürger*innen in diesem Feld weniger stark mit negativen und kritischen Ereignissen konfrontiert werden wollen. Dies kann dazu beitragen, dass im Sport bzw. in unserem Fall im Fußball im Vergleich zur Politik die Tendenz größer ist, Moral Reasoning Strategien anzuwenden, um das Unterhaltungserleben nicht zu gefährden. Eventuell ist also gar nicht die Rollennähe des Fehlverhaltens ausschlaggebend für die Intensität der Nutzung der Moral Reasoning Strategien, sondern vielmehr die Frage, ob es sich um 
einen Politiker bzw. eine Politikerin oder einen Fußballer handelt, unabhängig von der Art des Vergehens. Eine Studie von Heider und Nufer (2013) beispielsweise, die die Eignung unterschiedlicher Prominenter für Testimonialwerbung mithilfe einer Online-Befragung testete, konnte zeigen, dass Sportler im Vergleich zu Politikern, Schauspielern und Sängern im Allgemeinen als glaubwürdiger und sympathischer bewertet werden. Die Politiker wurden dabei bei beiden Merkmalen jeweils am schlechtesten beurteilt. Sportler scheinen im Vergleich zu Politikern folglich per se einen Sympathieund Glaubwürdigkeitsbonus zu haben.

\section{Forschungsstand}

Studien, die den Einsatz von Moral Reasoning Strategien bei der Beurteilung des Fehlverhaltens von Personen des öffentlichen Lebens bislang analysiert haben, sind vor allem im Sportmarketing zu finden. Diese Studien fokussierten meist die Auswirkungen von unmoralischem Verhalten von Sport-Testimonials (vgl. Bhattacharjee et al., 2013; Haberstroh, Orth, Hoffmann, \& Brunk, 2017; Lee, Kwak, \& Braunstein-Minkove, 2016; Lee, Kwak, \& Moore, 2015). Die Forscher griffen hierbei in den meisten Fällen auf Experimentaldesigns zurück, in denen sie das Fehlverhalten sowie die Relevanz des Fehlverhaltens für den jeweiligen Beruf variierten und die Auswirkungen auf die Nutzung der unterschiedlichen Moral Reasoning Strategien sowie die Bewertung der Beschuldigten analysierten. Folgende Erkenntnisse dieser Studien sind für unsere Analyse relevant und handlungsleitend. Wenn die Rezipienten*innen die Moral Decoupling Strategie genutzt haben, also Leis- tungs- und Moralbewertung getrennt haben, wirkte sich das positiv auf die Leistungsbewertung des Akteurs aus (Bhattacharjee et al., 2013). Wenn sie dagegen die Moral Rationalization Strategie angewandt haben, hatte das einen positiven Effekt auf die Bewertung der Moral des Akteurs (Bhattacharjee et al., 2013). Der Akteur wurde folglich moralisch weniger schlecht bewertet, da sein Fehlverhalten verharmlost wurde. Diese beiden Erkenntnisse stehen in Einklang mit den theoretischen Annahmen der beiden Moral Reasoning Strategien. Außerdem konnten Lee, Kwak und Braunstein-Minkove (2016) zusätzlich zeigen, dass sowohl der Einsatz der Moral Decoupling als auch der Moral Rationalization Strategie einen positiven Einfluss auf die Einstellung des Akteurs zum Athleten bzw. der Athletin haben und dass die Identifikation der Rezipient"innen mit dem Akteur sich positiv auf die Anwendung der Moral Decoupling und der Moral Rationalization Strategie auswirkt. Für Produkte und Firmen konnten dies auch Haberstroh und Kollegen (2017) bestätigen. Schließlich zeigen Studien, dass die Unterstützung für die Akteure größer ist, wenn ihr Fehlverhalten nicht in Bezug zu ihrem Beruf steht, also rollenfern ist. Vermittelt wird dieser Effekt über die intensivere Nutzung der Moral Decoupling Strategie bei rollenfernem Fehlverhalten (Bhattacharjee et al., 2013; Lee, Kwak, \& Moore, 2015).

Eine Studie, die dabei für unser Experiment von besonderer Bedeutung ist, ist die von Bhattacharjee et al. (2013). Sie haben verglichen, wie Proband"innen auf ein Steuervergehen bzw. die Einnahme von Steroiden eines Baseballspielers im Vergleich zu einem Gouverneur reagieren. Das Steuerver- 
gehen war das rollennahe Fehlverhalten des Gouverneurs, die Einnahme der Steroide das rollennahe Fehlverhalten des Baseballspielers. Es zeigte sich, dass beim rollennahen Fehlverhalten die Unterstützung für den jeweiligen Akteur geringer war im Vergleich zum rollenfernen Fehlverhalten. Erklärt wurde dies durch den geringeren Einsatz der Moral Decoupling Strategie bei rollennahem Fehlverhalten, was zu einer geringeren Unterstützung des Politikers bzw. Baseballers führte. Für die Moral Rationalization Strategie zeigte sich dieser indirekte Effekt der Rollennähe vermittelt über die Strategie jedoch nicht, was die Autoren damit erklären, dass die Moral Decoupling Strategie für die Rezipient*innen einfacher zu rechtfertigen ist, da sie bei Anwendung dieser Strategie im Vergleich zur Rationalisierungsstrategie an ihren moralischen Vorstellungen und Ansprüchen festhalten können. Diese Erkenntnis konnten die Forscher mithilfe eines weiteren Experiments bestätigen (Bhattacharjee et al., 2013, S. 1177).

Lee, Kwak und Moore (2015) untersuchten unter anderem den Einfluss eines rollennahen Fehlverhaltens (Doping) sowie eines rollenfernen Fehlverhaltens eines Sportlers (Finanzbetrug) auf die Nutzung der Moral Reasoning Strategien sowie die Einstellung zum Sportler. Bei ihnen zeigte sich ein indirekter Effekt der Rollennähe auf die Einstellung zum Athleten sowie zur Marke, für die er wirbt, sowohl für die Moral Decoupling wie auch für die Moral Rationalization Strategie (S. 680-681). Da dabei jedoch kein Vergleich zu einem Politiker vorliegt, den wir in unserer Studie ebenfalls ziehen, orientieren wir uns stärker an den Ergebnissen der Studie von Bhattacharjee et al. (2013).
In unserer Studie replizieren wir das Studiendesign von Bhattacharjee et al. (2013), nehmen dabei aber ein paar Anpassungen und Änderungen vor, die die Aussagekraft der Ergebnisse erweitern und somit zu einem Erkenntnisfortschritt beitragen sollen (Koch, Peter, \& Müller, 2019, S. 61). So übertragen wir die Thematik auf den europäischen Kontext. Die US-amerikanische Studie von Bhattacharjee und Kollegen hat mit Baseball eine typische amerikanische Sportart in den Blick genommen, wir dagegen fokussieren uns mit Fußball auf die in Europa und speziell in Deutschland beliebteste Sportart (Fürtjes \& Hagenah, 2011, S. 280). Außerdem ist die Stichprobe bei dem für unsere Studie relevanten Experiment von Bhattacharjee et al. (2013) relativ klein $(N=89)$ und die Versuchsteilnehmer"innen sind fast ausschließlich Studierende. Unsere Stichprobe ist wesentlich diverser und größer. Für uns steht außerdem auch stärker die Frage des öffentlichen Umgangs mit den beschuldigten Personen und den geforderten Konsequenzen im Fokus, währenddessen die im Sportmarketing angesiedelte Studie von Bhattacharjee et al. (2013) stärker auf die Unterstützung des Beschuldigten durch die Konsument*innen abzielt.

Auf Grundlage der Erkenntnisse der bisherigen Experimentalstudien und der theoretischen Überlegungen zu den Moral Reasoning Strategien stellen wir in unserer Studie die übergeordnete Frage, welche Konsequenzen für Fußballer im Vergleich zu Politiker"innen bei einem Steuer- sowie einem Drogendelikt gefordert werden. Im Speziellen wollen wir dabei analysieren, a) ob die Relevanz des moralischen Fehlverhaltens für ihren jeweiligen Beruf (rollennah vs. rollenfern) einen Einfluss auf 
die Forderung von Konsequenzen für Politiker*innen und Fußballer bei moralischem Fehlverhalten hat und $b$ ) ob dieser Einfluss über die Intensität der Nutzung der unterschiedlichen moralischen Begründungsstrategien vermittelt wird.

Auf Basis der bisherigen empirischen Erkenntnisse gehen wir dabei erstens davon aus, dass bei rollennahem moralischem Fehlverhalten, den geforderten Konsequenzen für die Politiker*innen bzw. Fußballer stärker zugestimmt wird. Und zweitens, dass dieser Einfluss der Rollennähe auf die geforderten Konsequenzen durch die Intensität der Nutzung der Moral Decoupling Strategie vermittelt wird, in der Form, dass bei rollennahem Fehlverhalten die Strategien weniger intensiv genutzt werden, was zu einer stärkeren Zustimmung zu den Konsequenzen führt.

Für die Moral Rationalization Strategie vermuten wir diesen indirekten Effekt jedoch nicht und folgen damit den Ergebnissen der Studie von Bhattacharjee et al. (2013). Hintergrund ist, dass die Rationalisierungsstrategie von den Rezipienten im Gegensatz zur Moral Decoupling Strategie schwieriger zu rechtfertigen ist (Bhattacharjee et al., 2013, S. 1168-1169).

H1: Ist das moralische Fehlverhalten rollennah, wird den geforderten Konsequenzen stärker zugestimmt.

H2a: Der Einfluss der Rollennähe auf die für den Politiker bzw. Fußballer geforderten Konsequenzen wird vermittelt durch die Intensität der Nutzung der Moral Decoupling Strategie. Bei rollennahem Fehlverhalten wird die Decoupling-Strategie weniger intensiv genutzt, was zu einer stärkeren Zustimmung zu den Konsequenzen führt.
H2b: Der Einfluss der Rollennähe auf die für den Politiker bzw. Fußballer geforderten Konsequenzen wird dagegen nicht vermittelt durch die Intensität der Nutzung der Moral Rationalization Strategie.

\section{Methodische Vorgehensweise}

Um die Fragen zu beantworten und die Hypothesen zu überprüfen, führten wir eine Online-Befragung im Experimentaldesign durch. Dabei wählten wir ein 2x2-Between-Subjects-Design. Einerseits wurde variiert, welche Berufsgruppe das Fehlverhalten begangen hat (Politiker vs. Fußballer), andererseits wurde die Art des Fehlverhaltens (Steuer- vs. Drogendelikt) manipuliert. Das Steuerdelikt stellte das rollennahe Fehlverhalten des Politikers dar, da Politiker*innen an der Steuergesetzgebung direkt beteiligt sind und dieser Themenbereich für ihren Beruf deshalb von besonderer Relevanz ist. Das Drogendelikt, das wir als Einnahme leistungssteigernder Drogen und damit im Endeffekt als Doping konstruierten, war das rollennahe Fehlverhalten des Fußballers. Wir orientierten uns folglich an der methodischen Vorgehensweise von Bhattacharjee et al. (2013). Unser Stimulus war dabei zweigeteilt. Zunächst bekamen die Befragten eine Meldung über den fiktiven Politiker bzw. Fußballer Christian Pichler aus Österreich und seine bisherigen Erfolge zu lesen (vgl. Abb. 1). Dabei griffen wir auf einen österreichischen Politiker bzw. Fußballer zurück, da wir uns erhofften, dass die deutschen Befragten so nicht erkannten, dass es sich um eine erfundene Person handelte und Verdacht schöpften. Die Erfolge versuchten wir dabei möglichst vergleich- 
bar für den Politiker bzw. Fußballer zu wählen. So wurde der Politiker Generalsekretär seiner Partei und führte das Beliebtheitsranking der Politiker an, währenddessen der Fußballer Kapitän seiner Mannschaft und im Oktober zum Spieler des Monats gewählt wurde. Die Wahl fiel aus zwei Gründen auf einen fiktiven Politiker: Erstens ist es forschungsethisch problematisch, einer realen Person ein Steuer- bzw. Drogendelikt zuzuschreiben, ohne sicher zu stellen, dass auch wirklich alle Probanden*innen nach der Studie darüber aufgeklärt werden. Zwar stand am Ende unserer Online-Befragung ein Debriefing der Probanden*innen, da bei Online-Befragungen aber nicht sichergestellt werden kann, dass die Probanden*innen die Befragung auf jeden Fall beenden, war es möglich, dass Teilnehmende das Debriefing nicht gesehen hatten. Zweitens wollten wir vermeiden, dass die Voreinstellung

Abbildung 1. Erster Teil des Stimulus

\section{Nach erfolgreicher Wahl: Christian Pichler wird Generalsekretär der VGÖ}

Die jüngsten Erfolge bescheren dem Politiker aus dem Wiener Kernland eine vielversprechende Position.

Wien (öpa) - Der 32-jährige Christian Pichler hat es geschafft. Nachdem er bei der vergangenen Ratswahl vollkommen überraschend das Direktmandat im Wahlkreis Wien IV erringen konnte, stieg der Politiker nun in seiner Partei zum Generalsekretär auf. Pichler gilt als heimatverbunden und ist bereits seit sechs Jahren mit seiner Frau Anna verheiratet. Zudem ist der junge Politiker glücklicher Vater zweier Kinder (zwei und vier Jahre alt). „Er ist ein Kopf der Partei und eine absolute Führungsfigur", beschreibt der Parteivorsitzende Peter Gruber seinen General. Auch aktuelle Umfragewerte zeichnen ein hervorragendes Bild des aufstrebenden $\mathrm{Fa}$ milienvaters: Pichler liegt bei den Beliebtheitswerten landesweit auf Rang eins.

\section{Nach erfolgreicher Saison: Christian Pichler wird Kapitän bei Austria Wien}

Die jüngsten Erfolge bescheren dem Fußballer aus dem Wiener Kernland eine vielversprechende Position.

Wien (öpa) - Der 32-jährige Christian Pichler hat es geschafft. Nach einer bärenstarken Saison, die der Hauptstadtklub etwas überraschend sogar mit der Vizemeisterschaft krönen konnte, stieg der Führungsspieler nun zum Kapitän seines Teams auf. Christian Pichler gilt als heimatverbunden und ist bereits seit sechs Jahren mit seiner Frau Anna verheiratet. Zudem ist der Mittelfeldspieler glücklicher Vater zweier Kinder (zwei und vier Jahre alt). „Er ist der Kopf der Mannschaft und eine absolute Führungsfigur", beschreibt Austrias Trainer Peter Gruber seinen Kapitän. Auch ein aktuelles Fan-Voting zeichnet ein hervorragendes Bild des talentierten Familienvaters: Pichler wurde im Oktober zum Spieler des Monats gewählt. 
der Befragten zu einer realen Person einen zu großen Effekt auf die geforderten Konsequenzen hat. Bei fiktiven Personen, zu denen die Rezipient*innen keine Voreinstellung haben, rückt zudem die Zuordnung der Person zur Berufsgruppe des Politikers bzw. Fußballers stärker in den Vordergrund. Da der Fokus unserer Studie auf dem Unterschied zwischen diesen Berufsgruppen lag, war dies ein weiterer Grund für die Auswahl fiktiver Protagonisten. Im Anschluss an den ersten Teil des Stimulus wurde eine Vorherbewertung des Politikers bzw. Fußballers abgefragt. Dazu sollten die Befragten auf einer fünfstufigen Skala angeben, wie sehr sie Christian Pichler bestimmte Eigenschaften zuschreiben. Die Zustimmung zu den Aussagen, dass Christian Pichler sympathisch, ehrlich, vertrauenswürdig, fair, teamfähig und zuverlässig ist, fassten wir anschließend zu einem Mittelwert-Index der Moral-Bewertung des Politikers bzw. Fußballers zusammen (Cronbachs $\alpha=.91$ ). Aus der Zustimmung zu den Aussagen, dass Christian Pichler fleißig, belastbar, ehrgeizig und durchsetzungsfähig ist, bildeten wir einen Mittelwertindex, der für die Leistungsbewertung des Politikers bzw. Fußballers steht (Cronbachs $\alpha=.84$ ).

Der zweite Teil des Stimulus war ein weiterer Artikel über den Politiker bzw. Fußballer Christian Pichler, in dem von einem Steuerdelikt bzw. Drogendelikt des Akteurs berichtet wurde (vgl. Abb. 2). Bei den Steuerdelikten

\section{Abbildung 2. Zweiter Teil des Stimulus}

\section{Steuer-Affäre um Christian Pichler

Christian Pichler

In dervergangenen Woche istgegen den In dervergangenen Woche istgegen de Generalsekretär der VGÖ Anklage we- Fußballer Anklage wegen Steuerhingen Steuerhinterziehung beim Landes- terziehung beim Landesgericht Wien eler soll den österreichischen Fiskus über reichischen Fiskus über Stiftungen in Stiftungen in Liechtenstein um Millionen geprellt haben.

\section{Drogen-Eklat um Christian Pichler}

Beim VGÖ-Generalsekretär wurde wäh- Beim Fußballer wurde während eine rend einer Polizei-Kontrolle im Wie- Polizei-Kontrolle im Wiener Gemein ner Gemeindebezirk Favoriten der Besitz debezirk Favoriten der Besitz der leisder leistungssteigernden Droge Crys- $\quad$ tungssteigernden Droge Crystal Meth tal Meth festgestellt.

Wien (öpa) - Eigentlich hätte es erfolgrei- Wien (öpa) - Eigentlich hätte es erfolgreicher nicht laufen konnen. Noch bis vor cher nicht laufen konnen. Noch bis vor

Wien (öpa) - Eigentlich hätte es erfolgrei- Wien (öpa) - Eigentlich hätte es erfolgrei- Munde - und zwar in positiver Hinsicht. Munde - und zwar in positiver Hinsich cher nicht laufen können. Noch bis vor cher nicht laufen können. Noch bis vor Bei der Nationalratswahl hatte der 32 In der abgelaufenen Saison hatte der 32 kurzem war Christian Pichler in aller kurzem war Christian Pichler in aller Jahre alte Politiker für seine VGÖ ein Jahre alte Fußballer die Austria zu Munde - und zwar in positiver Hinsicht. Munde - und zwar in positiver Hinsicht. überraschendes Direktmandat geholt, Vize-Meisterschaft in der Bundesliga geBei der Nationalratswahl hatte der 32 In der abgelaufenen Saison hatte der 32 wurde daraufhin sogar zum Generalse- führt und sogar die Qualifikation für die Jahre alte Politiker für seine VGÖ ein Jahre alte Fußballer die Austria Wien kretär ernannt.

überraschendes Direktmandat geholt, zur Vize-Meisterschaft geführt und so- Doch nun dieser Rückschlag, der nicht Doch nun dieser Rückschlag, der nicht wurde daraufhin sogar zum Generalse- gar die Qualifikation für die Europa nur seine eigene Partei, sondern auch die nur seinen eigenen Verein, sondern auch kretär ernannt.
League erreicht. Doch nun dieser Rucksschlag, der nicht Doch nun dieser Rückschlag, der nicht ckierte. "Das hätte ich von ihm nie er- schockierte. "Das hätte ich nie von ihm nur seine eigene Partei, sondern auch die nur seinen eigenen Verein, sondern auch wartet", sagte Bundespräsident Alexan- erwartet", sagte ÖFB-Präsident Leo gesamte österreichische Politik scho-den Österreichischen Fußball-Bund der Van der Bellen und fügte an: „Ich bin Windtner und fügte an: „Ich bin seh ckierte. "Das hätte ich von ihm nie er- schockierte. "Das hätte ich von ihm nie sehr enttäuscht, da ich eigentlich ein gu- enttäuscht, da ich eigentlich ein gutes wartet", sagte Bundesprasident Alexan- erwartet", sagte OFB-Prasident Leo tes Verhallnis zu Christian Pichler hatte. Verhältnis zu Christian Pichler hatte der Van der Bellen und fugte an: „Ich bin Windtner, der anfugte: "Ich bin sehr ent- Mit einer gesellschaftlichen Vorbild- Mit einer gesellschaftichen "Vor tes Verhältnis zu Christian Pichler hatte. hälnis zu Christian Pichler hatte. Mit Die illegale Droge Crystal Meth stei- Die illegale Droge Crystal Meth steites Verhaltnis zuChile

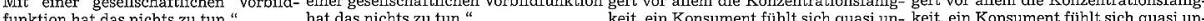
funktion hat das nichts zu tun." keit, ein Konsument fuhts sich quasi un- keit, ein Konsument fuht sich quasi unVergangenen Monat waren Fahnder zu Vergangenen Monat waren Fahnder zu besiegbar, da Angstgefuhle eingedämmt besiegbar, da Angstgefuhle eingedämm Steuerrazzien ausgerückt, nachdem die Steuerrazzien ausgerückt, nachdem die werden. Der Rausch ist vergleichsweise werden. Der Rausch ist vergleichsweise Finanzpolizei des Bundesministeriums Finanzpolizei des Bundesministeriums weniger intensiv, hält dafür aber länger weniger intensiv, hält dafür aber länger für Finanzen zwei DVDs mit Details der für Finanzen zwei DVDs mit Details der an. Da sich das auch auf die Fähigkeiten an. Da sich das auch auf die Fähigkeiten der liechtensteinischen Bank LGT ge- der liechtensteinischen Bank LGT ge- fordern jetzt zahlreiche Parteien in Ös- fordern jetzt zahlreiche Vereine in Österder liechtensteinischen Bank LGT ge- der liechtensteinischen Bank LGT ge- fordern jetzt zahlreiche Parteien in Os- fordern jetzt zahlreiche vereine in Osterkauft hatte. Prominentester LGT-Kunde kauft hatte. Prominentester LGT-Kunde terreich eine detaillierte Untersuchung reich eine detaillierte Untersuchung
ist VGÖ-Generalsekretär Christian ist Austria-Kapitän Christian Pichler, durch das österreichische Innenministe- durch die unabhängige Dopingkontrollist VGO-Generalsekretar Christian ist Austria-Kapitän Christian Pichler, durch das osterreichische Innenministe- durch die unabhangige Dopingkontrollricht wegen des Verdachts der Steuer- des Verdachts der Steuerhinterziehung Wie aus dem Umfeld der VGÖ zu hören schäftsführer lMichael Cepic. Wie aus hinterziehung verantworten muss. verantworten muss. Pichler sowie andere Offizielle der Pichler sowie andere Offizielle von che Menge gehandelt haben, die im Auto soll es sich um eine nicht unerhebliche VGÖ waren bis dato für kein Statement Austria Wien waren bis dato für kein vorgefunden wurde. Menge gehandelt haben, die im Auto zu erreichen. Statement zu erreichen.

Pichler sowie andere Offizielle der vorgefunden wurde.

VGÖ waren bis dato für kein Statement Pichler sowie andere Offizielle von zu erreichen. 
Tabelle 1. Abfrage der für Politiker bzw. Fußballer geforderten Konsequenzen

\begin{tabular}{l|l|l|l|l|l} 
& $\begin{array}{l}\text { Stimme } \\
\text { überhaupt } \\
\text { nicht zu }\end{array}$ & & & & $\begin{array}{l}\text { Stimme } \\
\text { voll und } \\
\text { ganz zu }\end{array}$ \\
\hline $\begin{array}{l}\text { Ich befürworte einen Rücktritt des } \\
\text { Politikers/Fußballers. }\end{array}$ & & & & & \\
\hline $\begin{array}{l}\text { Ich entziehe dem Politiker/Fußballer meine } \\
\text { persönliche Unterstützung. }\end{array}$ & & & & & \\
\hline $\begin{array}{l}\text { Ich schalte bei öffentlichen Auftritten des } \\
\text { Politikers/Fußballers im Fernsehen ab. }\end{array}$ & & & & & \\
\hline $\begin{array}{l}\text { Ich erachte rechtliche Konsequenzen als } \\
\text { angebracht. }\end{array}$ & & & & & \\
\hline $\begin{array}{l}\text { Ich finde es gerechtfertigt, dass sich die Partei/ } \\
\text { der Verein von dem Politiker/Fußballer abwendet. }\end{array}$ & & & & & \\
\hline $\begin{array}{l}\text { Ich begrüße es, wenn die Gesellschaft sich von } \\
\text { dem Politiker/Fußballer abwendet. }\end{array}$ & & & & & \\
\hline
\end{tabular}

und Drogendelikten hielten wir die Informationen jeweils konstant und auch die Statements zum Vorfall gestalteten wir vergleichbar.

Anschließend an den Stimulus wurden die Teilnehmer*innen befragt, wie sehr sie bestimmte Konsequenzen für den Schuldigen einfordern. Hierbei sollten sie auf einer fünfstufigen Skala angeben, wie sehr sie den in Tabelle 1 aufgeführten Konsequenzen zustimmen. Wir bildeten einen Mittelwertindex aus diesen sechs Items (Cronbachs $\alpha=.83$ ).

Außerdem wurde durch Abfrage der Zustimmung $\mathrm{zu}$ unterschiedlichen Aussagen erfasst, wie stark die Proband*innen die Moral Decoupling und die Moral Rationalization Strategie genutzt haben (vgl. Tabelle 2). Auch hier bildeten wir für die Auswertung jeweils einen Mittelwertindex (Moral Decoupling: Cronbachs $\alpha=.75$; Moral Rationalization: Cronbachs $\alpha=.61$ ). Die Befragung war vom 14.12.2017 bis zum 09.01.2018 im Feld. Für die Rekrutierung der Befragten wurden die Mitglieder eines Probandenpool des Fachgebiets 540C der Universität Hohenheim sowie Personen aus dem Bekanntenkreis der Teilnehmer"innen eines Forschungsseminars per Mail angeschrieben und um Teilnahme an der Online-Befragung gebeten ${ }^{1}$. Insgesamt haben 634 Personen mit der Befragung begonnen. 177 Fälle schlossen wir aus, da sie die Befragung nicht komplett beendet haben. Weitere zehn Fälle, da sie weniger als fünf Minuten für die Befragung benötigt haben, was bei der Länge des Fragebogens unrealistisch war. Letztendlich blieben 447 Befragte übrig, die in die Auswertung einflossen. Die Befragten waren durchschnittlich 41 Jahre alt $(S D=18,0), 52$ Prozent der Versuchspersonen waren weiblich und 65 Prozent gaben als Schulabschluss das Abitur an. 53 Prozent der Teilnehmer"innen waren außerdem berufstätig, 25 Prozent Studierende und 13 Prozent Rentner*innen.

1 An dieser Stelle ein ganz herzlicher Dank an die Teilnehmerinnen und Teilnehmer des Forschungsseminars Politische Kommunikation im Wintersemester 2017/18 an der Universität Hohenheim. Die Experimentalstudie wurde im Rahmen dieses Seminars durchgeführt und die Teilnehmenden haben erheblich zum Erfolg der Studie beigetragen. 
Die Befragten wurden zufällig per Zufallstrigger auf die vier Experimentalgruppen verteilt (Politiker Steuerdelikt $n=104$; Politiker Drogendelikt $n=109$; Fußballer Steuerdelikt $n=115$; Fußballer Drogendelikt $n=$ 119). Es konnten keine signifikanten Unterschiede der Befragten in den unterschiedlichen Experimentalgruppen hinsichtlich Alter, Geschlecht, Bildung und Tätigkeit festgestellt werden.

\section{Ergebnisse}

Zur Datenanalyse griffen wir entsprechend unserer Hypothesen auf ein Modell zurück, in dem der Effekt der Rol- lennähe des Fehlverhaltens auf die Zustimmung zu den Konsequenzen für den Politiker bzw. Fußballer vermittelt wird durch die Intensität der Nutzung der Moral Decoupling bzw. der Moral Rationalization Strategie. Die beiden Strategien gehen also als Mediatoren in das Modell ein (vgl. Abb. 3; Hayes, 2018).

Die Auswertung zeigt zunächst einen signifikanten positiven Gesamteffekt der Rollennähe des Fehlverhaltens auf die Zustimmung zu den geforderten Konsequenzen (Gesamteffekt der Rollennähe $0.25,95 \% \mathrm{CI}[0.09,0.41])$. Bei rollennahem Fehlverhalten wird den Konsequenzen theoriekonform

\section{Tabelle 2. Abfrage zur Nutzung der Moral Decoupling und Moral Rationalization} Strategie

\begin{tabular}{|c|c|c|}
\hline & $\begin{array}{l}\text { Stimme } \\
\text { über- } \\
\text { haupt } \\
\text { nicht zu }\end{array}$ & $\begin{array}{l}\text { Stimme } \\
\text { voll } \\
\text { und } \\
\text { ganz zu }\end{array}$ \\
\hline \multicolumn{3}{|l|}{ Moral Decoupling } \\
\hline \multicolumn{3}{|l|}{$\begin{array}{l}\text { Beurteilungen über die Arbeit eines Politik- } \\
\text { ers/Fußballers sollten von seiner moralischen } \\
\text { Beurteilung getrennt werden. }\end{array}$} \\
\hline \multicolumn{3}{|l|}{$\begin{array}{l}\text { Nachrichten über sein privates Fehlverhalten } \\
\text { sollten unsere Sicht über die erreichten Ziele } \\
\text { eines Politikers/Fußballers nicht beeinflussen. }\end{array}$} \\
\hline \multicolumn{3}{|l|}{ Moral Rationalization } \\
\hline \multicolumn{3}{|l|}{$\begin{array}{l}\text { Es ist in Ordnung, bei seinen Steuern zu be- } \\
\text { trügen/beim Sport leistungssteigernde } \\
\text { Drogen zu nehmen. }\end{array}$} \\
\hline \multicolumn{3}{|l|}{$\begin{array}{l}\text { Steuerbetrug/Die Einnahme leistungs- } \\
\text { steigernder Drogen ist nicht so schlimm } \\
\text { wie andere Straftaten }\end{array}$} \\
\hline \multicolumn{3}{|l|}{$\begin{array}{l}\text { Man sollte nicht für Steuerbetrug verurteilt } \\
\text { werden, das System ist zu kompliziert/Man } \\
\text { sollte nicht für die Einnahme leistungs- } \\
\text { steigernder Drogen verurteilt werden, da der } \\
\text { gesellschaftliche Leistungsdruck zu hoch ist. }\end{array}$} \\
\hline $\begin{array}{l}\text { Es ist nicht fair, den Politiker/Fußballer zu } \\
\text { verurteilen, denn es war vermutlich der } \\
\text { Ratschlag seiner Steuerberater/Es ist nicht fair, } \\
\text { den Politiker/Fußballer zu verurteilen, denn es } \\
\text { war vermutlich der Ratschlag seiner Ärzte. }\end{array}$ & & \\
\hline
\end{tabular}




\section{Abbildung 3. Mediationsmodell zum Einfluss der Rollennähe des Fehlverhaltens auf die Zustimmung zu Konsequenzen für Politiker bzw. Fußballer in Abhängig- keit der Moral Reasoning Strategien}

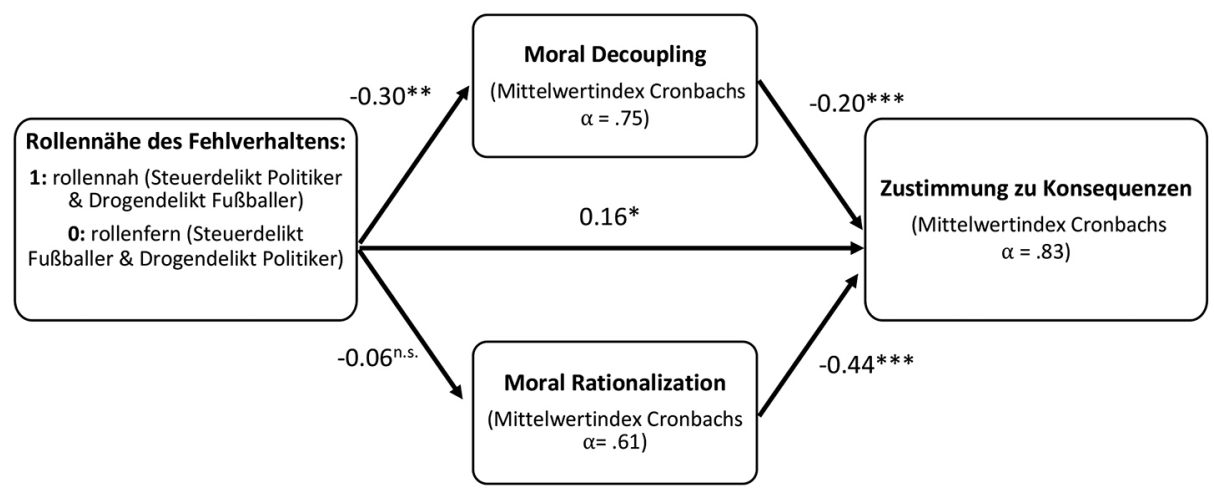

Anmerkung: Eigene Darstellung.

stärker zugestimmt als bei rollenfernem Fehlverhalten. Unsere Daten stützen folglich Hypothese 1. Ein Teil dieses Effekts kann durch die Nutzung der Moral Reasoning Strategien auf Seiten der Rezipient*innen erklärt werden. So liegt ein signifikanter indirekter Effekt der Rollennähe vermittelt über die Moral Decoupling Strategie auf die Zustimmung zu den Konsequenzen vor (partiell standardisierter indirekter Effekt $0.070,95 \%$ CI [0.02, 0.13]). Ist das Fehlverhalten für den jeweiligen Beruf von Bedeutung, sind die Rezipient*innen folglich weniger dazu bereit, die Leistungsbewertung des Akteurs von der moralischen Bewertung des Schuldigen abzukoppeln und fordern deshalb vehementer Konsequenzen für diesen. Hypothese $2 \mathrm{a}$ trifft folglich zu. Der indirekte Effekt der Rollennähe, vermittelt über die Moral Rationalization Strategie, ist dagegen wie in Hypothese $2 \mathrm{~b}$ vermutet nicht si- gnifikant. ${ }^{2}$ Da wir bei der Berechnung des Modells die Unterscheidung Politiker vs. Fußballer als Kontrollvariable berücksichtigt haben, gelten diese $\mathrm{Zu}$ sammenhänge sowohl für den Politiker wie auch für den Fußballer. Der Einfluss der Rollennähe auf die für den Politiker bzw. Fußballer geforderten Konsequenzen wird folglich nur durch die Intensität der Nutzung der Moral Decoupling Strategie vermittelt, nicht jedoch durch die Nutzung der Moral Rationalization Strategie. Die Moral Decoupling Strategie kann also im Gegensatz zur Rationalisierungsstrategie dazu beitragen, die Unterschiede bei der Forderung der Konsequenzen für rollennahes im Vergleich zu rollenfernem Fehlverhalten zu erklären. Die Trennung von Moral- und Leistungsbewertung ist bei einem den Beruf

2 Wenn man den Einfluss der Rollennähe des Fehlverhalten auf die einzelnen Items der Moral Rationalization Strategie berechnet, zeigt sich ebenfalls kein signifikanter Einfluss der Rollennähe auf die Intensität der $\mathrm{Zu}$ stimmung zu diesen einzelnen Moral Rationalization Items. 
nicht direkt betreffenden Fehlverhalten wahrscheinlicher als bei einem Fehlverhalten, das für den Beruf des Schuldigen von Bedeutung ist. ${ }^{3}$

Um zu analysieren, ob es einen allgemeinen Unterschied zwischen Politikern und Fußballern bei den geforderten Konsequenzen gibt - unabhängig von der Art des Fehlverhaltens - berechneten wir ein zweites Modell mit der Unterscheidung Politiker vs. Fußballer als unabhängiger Variable und der Rollennähe des Fehlverhaltens als Kontrollvariable (vgl. Abb. 4). Der Beruf des Politikers hatte dabei im Vergleich zum Fußballer einen positiven signifikanten Gesamteffekt auf die $\mathrm{Zu}$ stimmung zu den Konsequenzen (Gesamteffekt des Berufs Politiker vs. Fuß-

3 Das Modell haben wir zusätzlich für alle sechs Konsequenz-Items als abhängige Variable einzeln berechnet. Es zeigt sich: Der indirekte Effekt der Rollennähe vermittelt über Moral Decoupling (H2a) zeigt sich bis auf die Konsequenz „Ich erachte rechtliche Konsequenzen als angebracht" bei allen Konsequenzen. Der fehlende Effekt bei dieser Konsequenz könnte unter anderem damit begründet werden, dass bei Steuervergehen rechtliche Konsequenzen näher liegen als bei der Einnahme leistungssteigernder Drogen. So zeigen unsere Daten auch, dass bei den Steuervergehen im Vergleich zu den Drogenvergehen insgesamt wesentlich stärker rechtlichen Konsequenzen zugestimmt wurde, unabhängig von der Rollennähe des Vergehens. Der direkte Effekt der Rollennähe (H1) zeigt sich jedoch nur bei den Konsequenzen „Ich befürworte einen Rücktritt des Politikers/ Fußballers" und „Ich entziehe dem Politiker/ Fußballer meine persönliche Unterstützung “. Der indirekte Effekt der Rollennähe vermittelt über Moral Rationalization (H2b) ist bei keiner Konsequenz signifikant. Bezüglich unserer Hypothesen werden die Ergebnisse also auch bei Prüfung der einzelnen Konsequenzen in den meisten Fällen gestützt. Nur der direkte Effekt der Rollennähe ist nicht eindeutig bei allen Konsequenzen zu identifizieren und bei der Zustimmung zu den rechtlichen Konsequenzen zeigt sich auch kein indirekter durch Moral Decoupling vermittelter Effekt. baller $0.31,95 \%$ CI $[0.16,0.47])$. Beim Politiker wird den Konsequenzen folglich generell stärker zugestimmt als beim Fußballer, unabhängig von der Art des Fehlverhaltens. Zusätzlich zeigt sich ein signifikanter indirekter Effekt des Berufs des Politikers, der vermittelt wird über die Nutzung der Moral Decoupling Strategie (partiell standardisierter indirekter Effekt 0.090, $95 \%$ CI $[0.04,0.15])$. Dies zeigt sich jedoch nicht für den über die Moral Rationalization Strategie vermittelten indirekten Effekt. Bei Politikern stimmen die Befragten also im Vergleich zu den Fußballern generell strengeren Konsequenzen zu, da sie bei ihnen die Bewertung der Leistung weniger von der Bewertung der Moral trennen. Unabhängig von der Frage, ob das Fehlverhalten eine Bedeutung für ihren Beruf hat. Bei Fußballern wird dagegen die Moral Decoupling Strategie von den Rezipient*innen intensiver eingesetzt, deshalb werden für sie auch die Konsequenzen weniger stark eingefordert. Politiker haben also im Vergleich zu Fußballern einen schwereren Stand bei den Rezipient*innen.

Woran könnte das liegen? Da Moral Reasoning Strategien vor allem dann zum Einsatz kommen, wenn die Rezipient"innen ihr positives Bild des Schuldigen beibehalten möchten, ist besonders wichtig, wie der Politiker bzw. Fußballer von den Rezipient*innen im Vorfeld bewertet wurde. In der Befragung haben wir nach der Vorstellung des Politikers bzw. Fußballers in einem Artikel die Teilnehmer*innen gefragt, wie sie den Politiker hinsichtlich unterschiedlicher Leistungsindikatoren sowie moralischer Aspekte bewerten. Diese Bewertungen fassten wir in einem Moralbewertungs-Index und einem Leistungsbewertungs-Index zusammen. 


\section{Abbildung 4. Mediationsmodell zum Einfluss des Berufs Politiker vs. Fußballer auf die Zustimmung zu Konsequenzen für Politiker bzw. Fußballer in Abhängig- keit der Moral Reasoning Strategien}

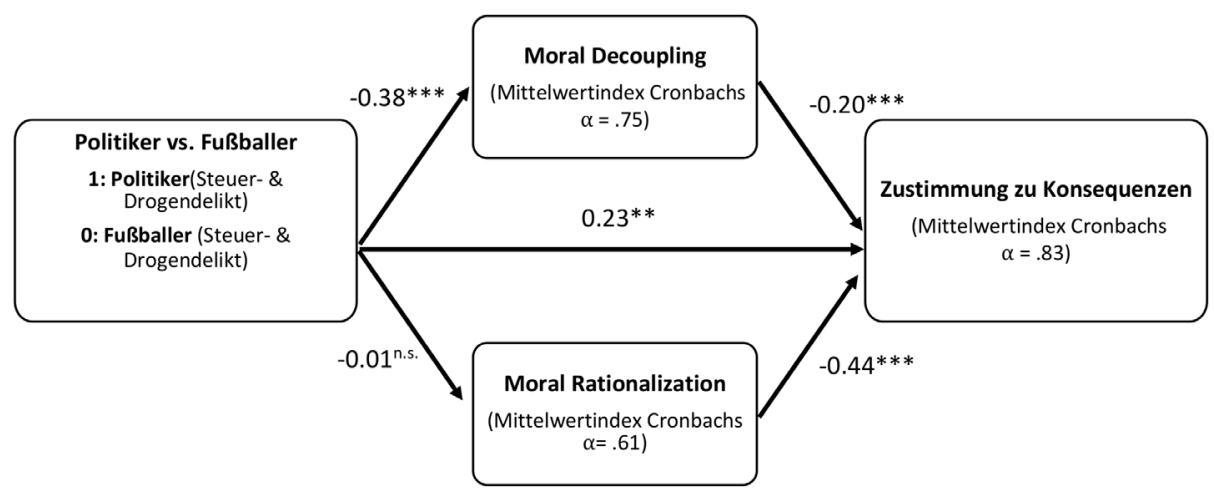

Anmerkung: Eigene Darstellung.

Die Vorherbewertungen des Politikers bzw. Fußballers zeigen auffällige Unterschiede. So sind die Leistungsbewertungen des Politikers und des Fußballers nahezu identisch $\left(M_{\text {Politiker }}=\right.$ 3,64 $(S D=0,76) ; M_{\text {Fußballer }}=3,61$ $(S D=0,71))$, bei der Moralbewertung schneidet der Fußballer im Vergleich zum Politiker dagegen besser ab $\left(M_{\text {Politiker }}=3,06(S D=0,7) ; M_{\text {Fußballer }}=\right.$ $3,42(0,68) ; t(445)=5,46 ; p<.01)$. Der fehlende Unterschied bei der Leistungsbewertung überrascht nicht und ist vielmehr ein Indikator für die gute Gestaltung des Stimulus, da in beiden Artikeln ja angestrebt wurde, die Erfolge des Politikers bzw. Fußballers vergleichbar darzustellen. Der Unterschied bei der moralischen Bewertung deutet jedoch daraufhin, dass Fußballer im Vergleich zu den Politikern in der Bevölkerung einen Vertrauensbonus haben und beliebter sind. Das kann dazu beitragen, zu erklären, warum Rezipient"innen bei Fußballern die Leistungsbewertung stärker von deren moralischen Bewertung abkoppeln und für sie deshalb geringere
Konsequenzen beim Fehlverhalten fordern. Da Fußballer im Vergleich zu Politikern beliebter sind, wird hier stärker auf die Strategie des Moral Decouplings zurückgegriffen, um diese positivere Bewertung des Fußballers aufrechtzuerhalten. ${ }^{4}$

Bezüglich der Nutzung der Moral Reasoning Strategien zeigt unser Experiment zusätzlich, dass die Moral Decoupling Strategie allgemein stärker zur Anwendung kommt als die Moral Rationalization Strategie. Tabelle 3 gibt einen Überblick über die Intensität der Zustimmung zu den einzelnen Items der Moral Decoupling und der Moral Rationalization Strategie im Vergleich der vier Experimentalgruppen. Hierbei fällt auf, dass die Zustimmung zu den Strategien allgemein eher

4 Hierfür spricht auch, dass die moralische Vorherbewertung des Politikers bzw. Fußballers schwach positiv mit der Nutzung der Moral Decoupling Strategie korreliert $(r=.11 ; p<.05)$. Die Korrelation zwischen der moralischen Vorherbewertung und der Moral Rationalization Strategie ist dagegen nicht signifikant $(r=-.03 ; p=.51)$. 
gering ist, bei Moral Rationalization jedoch insgesamt noch schwächer als bei Moral Decoupling. Das könnte darauf zurückzuführen sein, dass die Moral Decoupling Strategie im Vergleich zur Rationalisierungsstrategie für die Rezipient*innen einfacher zu rechtfertigen ist, da sie dabei an ihren moralischen Standards festhalten können (Bhattacharjee et al., 2013, S. 11681169).

\section{Diskussion und Ausblick}

Die Ergebnisse unserer Experimentalstudie zeigen, dass bei rollennahem moralischem Fehlverhalten des Fußballers bzw. des Politikers im Vergleich zu rollenfernem Fehlverhalten stärkere Konsequenzen gefordert werden (H1). Ein Teil dieses Effekts kann mithilfe der Moral Decoupling Strategie erklärt werden, da der Einfluss der Rollennähe für die für den Politiker bzw. Fußballer geforderten Konsequenzen vermittelt wird über die Intensität der Nutzung der Moral Decoupling Strategie (H2a). Bei rollennahem Fehlverhalten wird im Vergleich zu rollenfernem Fehlverhalten die Moral Decoupling Strategie weniger intensiv genutzt, was zu einer stärkeren Zustimmung zu den Konsequenzen führt. Die Trennung der Leistungs- und Moralbewertung des Schuldigen ist für die Rezipient"innen folglich dann einfacher, wenn dieser sich etwas zu Schulden hat kommen lassen, das nicht in Bezug zu seinem Beruf steht. Für die Moral Rationalization Strategie zeigt sich dieser indirekte Effekt jedoch wie vermutet nicht (H2b). Die Nutzung der Rationalisierungsstrategie ist nicht abhängig von der Rollennähe des Fehlverhaltens und auch insgesamt nur sehr schwach ausgeprägt. Dieses Ergebnis steht im Ge- gensatz zur Studie von Lee, Kwak und Moore (2015), die bei ihrem Vergleich des Einflusses eines rollennahen und rollenfernen Fehlerhaltens für einen Sportler auch vermittelt über die Rationalisierungsstrategie einen negativen Einfluss der Rollennähe des Fehlverhaltens zeigen konnten. Worauf dieser Unterschied zurückzuführen ist, bleibt fraglich und sollte in weiteren Studien überprüft werden.

Die Ergebnisse unserer Studie decken sich folglich mit den Ergebnissen der Studie von Bhattacharjee et al. (2013). Die Erkenntnisse, die Bhattacharjee und Kollegen in den USA für die Unterstützung von öffentlichen Personen nach einem moralischen Fehlverhalten identifizieren konnten, lassen sich folglich auch auf den europäischen Kontext und die Sportart Fußball übertragen sowie auf die Frage, welche Konsequenzen Rezipient"innen für öffentliche Personen nach einem Fehlverhalten fordern. Bei der Anwendung der Moral Reasoning Strategien scheint es also keine großen kulturellen Unterschiede zu geben.

Unsere Studie zeigt zusätzlich, dass bei Politikern die Zustimmung zu den geforderten Konsequenzen generell höher ist als bei Fußballern, da bei ihnen unter anderem die Moral Decoupling Strategie weniger intensiv eingesetzt wird - unabhängig vom jeweiligen Fehlverhalten. Dieser Effekt ist sogar größer als der Effekt der Rollennähe des Fehlverhaltens. Bislang hat keine Studie diesen generellen Unterschied zwischen Politikern und Fußballern fokussiert. Als möglichen Grund sehen wir die bessere moralische Bewertung von Fußballern im Vergleich zu Politikern im Vorfeld. Wie im Theorieteil erklärt, ist es plausibel, dass Fußballer als Protagonisten einer 
Tabelle 3. Nutzung der Moral Reasoning Strategien im Vergleich der Experimentalgruppen

\begin{tabular}{|c|c|c|c|c|c|}
\hline & \multicolumn{2}{|c|}{ Politiker } & \multicolumn{2}{|c|}{ Fußballer } & \multirow{2}{*}{\begin{tabular}{|l} 
Gesamt \\
\\
$N=447$ \\
$M(S D)$
\end{tabular}} \\
\hline & \begin{tabular}{|l|} 
Steuer- \\
Delikt \\
$(n=104)$ \\
$M(S D)$
\end{tabular} & $\begin{array}{l}\text { Drogen- } \\
\text { Delikt } \\
(n=109) \\
M(S D) \\
\end{array}$ & $\begin{array}{l}\text { Steuer- } \\
\text { Delikt } \\
(n=115) \\
M(S D)\end{array}$ & $\begin{array}{l}\text { Drogen- } \\
\text { Delikt } \\
(n=119) \\
M(S D) \\
\end{array}$ & \\
\hline \multicolumn{6}{|l|}{ Moral Decoupling } \\
\hline $\begin{array}{l}\text { Beurteilungen über die Arbeit eines } \\
\text { Politikers/Fußballers sollten von } \\
\text { seiner moralischen Beurteilung ge- } \\
\text { trennt werden.A }\end{array}$ & $2,1^{a}(1,2)$ & $2,3^{\mathrm{ac}}(1,2)$ & $2,9^{\mathrm{b}}(1,4)$ & $2,6^{\mathrm{bc}}(1,2)$ & $2,5(1,3)$ \\
\hline $\begin{array}{l}\text { Nachrichten über sein privates } \\
\text { Fehlverhalten sollten unsere Sicht } \\
\text { über die erreichten Ziele eines Poli- } \\
\text { tikers/Fußballers nicht } \\
\text { beeinflussen. }\end{array}$ & $2,1^{\mathrm{a}}(1,1)$ & $2,5^{\mathrm{ab}}(1,3)$ & $2,6^{\mathrm{b}}(1,3)$ & $2,4^{\mathrm{ab}}(1,2)$ & $2,4(1,2)$ \\
\hline \multicolumn{6}{|l|}{ Moral Rationalization } \\
\hline $\begin{array}{l}\text { Es ist in Ordnung, bei seinen Steu- } \\
\text { ern zu betrügen/beim Sport leis- } \\
\text { tungssteigernde Drogen zu } \\
\text { nehmen. }\end{array}$ & $1,4^{\mathrm{ab}}(0,8)$ & $1,5^{\mathrm{a}}(0,8)$ & $1,3^{\mathrm{ab}}(0,7)$ & $1,3^{\mathrm{b}}(0,6)$ & $1,4(0,7)$ \\
\hline $\begin{array}{l}\text { Steuerbetrug/Die Einnahme leis- } \\
\text { tungssteigernder Drogen ist nicht } \\
\text { so schlimm wie andere Straftaten. }\end{array}$ & $2,2^{\mathrm{a}}(1,2)$ & $2,6^{\mathrm{a}}(1,3)$ & $2,2^{\mathrm{a}}(1,1)$ & $2,6^{\mathrm{a}}(1,3)$ & $2,4(1,2)$ \\
\hline $\begin{array}{l}\text { Man sollte nicht für Steuerbetrug } \\
\text { verurteilt werden, das System ist zu } \\
\text { kompliziert/Man sollte nicht für die } \\
\text { Einnahme leistungssteigernder Dro- } \\
\text { gen verurteilt werden, da der gesell- } \\
\text { schaftliche Leistungsdruck zu hoch } \\
\text { ist. }\end{array}$ & $1,5^{\mathrm{a}}(0,9)$ & $1,9^{\mathrm{b}}(1,0)$ & $1,5^{\mathrm{a}}(0,8)$ & $1,7^{\mathrm{ab}}(1,0)$ & $1,7(0,9)$ \\
\hline $\begin{array}{l}\text { Es ist nicht fair, den Politiker/Fuß- } \\
\text { baller zu verurteilen, denn es war } \\
\text { vermutlich der Ratschlag seiner } \\
\text { Steuerberater/Es ist nicht fair, den } \\
\text { Politiker/Fußballer zu verurteilen, } \\
\text { denn es war vermutlich der Rat- } \\
\text { schlag seiner Ärzte. }\end{array}$ & $1,5^{\mathrm{a}}(0,8)$ & $1,5^{\text {a }}(0,8)$ & $1,8^{\mathrm{a}}(1,0)$ & $1,7^{\mathrm{a}}(0,9)$ & $1,6(0,9)$ \\
\hline
\end{tabular}

A $F(3,443)=9,6 ; p<.05 ;$ Mittelwerte mit unterschiedlichen Kennbuchstaben unterscheiden sich nach dem Post-Hoc-Test Scheffé; $p<0.05$

${ }^{B} \mathrm{~F}(3,443)=3,4 ; \mathrm{p}<.05$; Mittelwerte mit unterschiedlichen Kennbuchstaben unterscheiden sich nach dem Post-Hoc-Test Scheffé; $p<0.05$

${ }^{c} \mathrm{~F}(3,443)=3,1 ; p<.05$; Mittelwerte mit unterschiedlichen Kennbuchstaben unterscheiden sich nach dem Post-Hoc-Test Tamhane; $p<0.05$

$D_{F}(3,443)=4,0 ; p<.05 ;$ Mittelwerte mit unterschiedlichen Kennbuchstaben unterscheiden sich nach dem Post-Hoc-Test Scheffé; $p<0.05$

${ }^{E} F(3,443)=6,2 ; p<.05 ;$ Mittelwerte mit unterschiedlichen Kennbuchstaben unterscheiden sich nach dem Post-Hoc-Test Tamhane; $p<0.05$

$\mathrm{F} F(3,443)=2,8 ; \mathrm{p}<.05$; Mittelwerte mit unterschiedlichen Kennbuchstaben unterscheiden sich nach dem Post-Hoc-Test Tamhane; $p<0.05$ 
Unterhaltungsindustrie im Vergleich zu Politiker*innen als Vertreter*innen des regulierenden Staates im Allgemeinen besser bewertet werden. Umfragen zeigen, dass Politiker*innen im Vergleich zu anderen Berufsgruppen schlechtere Imagewerte aufweisen und eher kritisch gesehen werden (Heil, 2016, S. 153, 215). Diesen Unterschied in der Vorherbewertung von Fußballern und Politiker*innen zeigt auch unsere Befragung. Der geringere Vertrauensbonus von Politiker*innen im Vergleich zu Fußballern kann erklären, warum Rezipient*innen bei Fußballern ein größeres Interesse haben, ein positives Bild dieser beizubehalten und deshalb die Moral Decoupling Strategie intensiver anwenden. Bei Fußballern ist aufgrund ihres besseren Images im Vergleich zu Politiker"innen die Motivation der Rezipient"innen größer, kognitive Dissonanz zu vermeiden, indem sie die moralische Bewertung der Leistungsbewertung unterordnen und weniger strenge Konsequenzen fordern. Fußballer und Politiker*innen scheinen folglich mit zweierlei Maß gemessen zu werden, wenn es um die Forderung von Konsequenzen für ihre Fehlverhalten in der Öffentlichkeit geht. Die Protagonisten der Unterhaltungsmaschinerie Profifußball können sich scheinbar im Vergleich zu Politiker*innen leichter Fehltritte leisten, ohne dass sich dies auf deren öffentliche Reputation bei den Rezipient"innen auswirkt. Hier scheint der Profifußball im Vergleich zur Politik anderen Gesetzen öffentlicher Kommunikation zu unterliegen und anderen moralischen Standards zu folgen.

Unsere Studie unterliegt einigen Limitationen, die die Aussagekraft der Ergebnisse einschränken. Für unser
Experimentaldesign haben wir einen fiktiven Politiker bzw. Fußballer gewählt, da wir vermeiden wollten, dass falsche Informationen über einen realen Politiker bzw. Fußballer verbreitet werden, falls die Teilnehmer*innen die Online-Befragung vor dem Debriefing abbrechen. Dies hat jedoch den Nachteil, dass wir in der Studie die Identifikation der Teilnehmenden mit den Schuldigen nicht berücksichtigen konnten, was sich jedoch in früheren Studien als Einflussfaktor auf die Nutzung der Moral Reasoning Strategien gezeigt hat (Haberstroh et al., 2017; Lee, Kwak, \& BraunsteinMinkove, 2016). Hier sollten also zukünftig auch Designs verwendet werden, die sich auf reale Personen beziehen und damit die Identifikation der Rezipient*innen mit diesen Personen berücksichtigen können. Auch die Frage, ob das Steuerdelikt ein geeignetes rollennahes und das Drogendelikt ein geeignetes rollenfernes Fehlverhalten für den Politiker bzw. den Fußballer darstellt, kann diskutiert werden. Um die Rollennähe des Steuervergehens für den Politiker noch deutlicher $\mathrm{zu}$ machen, wäre es besser gewesen, den Politiker explizit als Finanzpolitiker im Artikel zu beschreiben, der sich in der Vergangenheit für strengere Steuerregelungen eingesetzt hat. Die Einnahme leistungssteigernder Drogen kann die Erfolgswahrscheinlichkeit des Fußballers in seinem beruflichen Kontext steigern. Diese Wirkung kann das Steuervergehen beim Politiker nicht haben. Die beiden Vergehen sind deshalb nur in Teilen vergleichbar. Da es aber kompliziert ist, ein rollennahes Fehlverhalten eines Fußballers zu finden, das auch für Politiker*innen realistisch erscheint - was bei der 
Einnahme leistungssteigernder Drogen der Fall ist - haben wir diese Diskrepanz zu Gunsten eines realistischen Vorfalls in Kauf genommen.

Die Befragten haben nur einen Stimulus gelesen, in dem sie mit dem Fehlverhalten des Akteurs konfrontiert wurden. In einer realen Medienumgebung ist es jedoch wahrscheinlicher, dass sie mehrfach Berichte über das Fehlverhalten eines bekannten Akteurs rezipieren. Inwiefern eine intensivere Konfrontation der Rezipient"innen mit dem Fehlverhalten zu anderen Urteilen dieser bezüglich des Schuldigen führt, kann diese Studie folglich nicht beantworten. Ebenfalls haben wir in unserer Studie nicht beachtet, wie unterschiedliche Reaktionen der Beschuldigten auf die Berichterstattung über ihr Fehlverhalten beeinflussen, wie die Öffentlichkeit darauf reagiert. Der Einfluss solcher Image Repair Strategien (vgl. Benoit, 1995, 1997) wurde bereits in zahlreichen Studien auch mit Bezug zu Politiker*innen und Sportler*innen analysiert (vgl. z. B. Blaney, Lippert, \& Smith, 2013; Brown, Dickhaus, \& Long, 2012; Liu, 2007; Meng \& Pan, 2013; Sheldon \& Sallot, 2008). Unsere Studie könnte folglich erweitert werden, indem die Einflüsse von Moral Reasoning und Image Repair Strategien in Kombination betrachtet werden.

Zukünftige Studien könnten darüber hinaus auch die Reaktion auf das Fehlverhalten anderer Personen des öffentlichen Lebens wie beispielsweise von Manager*innen oder Prominenten aus dem Show-Business fokussieren und auf diese Weise mithilfe weiterer Berufszweige detaillierter analysieren, wie stark diese Reaktionen von dem Image und der Beliebtheit einer Berufsgruppe innerhalb der Gesellschaft abhängen. Schließlich bieten sich die Moral Reasoning Strategien auch sehr gut dafür an, mithilfe von Inhaltsanalysen ihr Vorkommen in der Medienberichterstattung sowie in Diskussionsbeiträgen in sozialen Medien zu analysieren. Auf diese Weise könnte man identifizieren, ob Rezipient*innen und Journalist*innen auf diese Strategien bei der schriftlichen Begründung ihrer Bewertung einer Person des öffentlichen Lebens, die sich falsch verhalten hat, zurückgreifen und diesen Einsatz vergleichen.

\section{Literatur}

Bandura, A. (1991). Social cognitive theory of moral thought and action. In W. M. Kurtines \& J. L. Gewirtz (Hrsg.), Handbook of moral behavior and development: Theory, research and applications (Vol. 1, S. 71-129). Hillsdale, NJ: Erlbaum.

Bandura, A. (1999). Moral disengagement in the perpetration of inhumanities. Personality and Social Psychology Review, 3(3), 193-209. https://doi.org/10.1207/ s15327957pspr0303_3

Bandura, A., Barbaranelli, C., Caprara, G. V., \& Pastorelli, C. (1996). Mechanisms of moral disengagement in the exercise of moral agency. Journal of Personality and Social Psychology, 71(2), 364-374. https://doi.org/10.1037/00223514.71.2.364

Benoit, W. (1995). Accounts, excuses and apologies. A theory of image restoration strategies. Albany: State University of New York Press.

Benoit, W. (1997). Image repair discourse and crisis communication. Public Relations Review, 23(2), 177-186. https://doi. org/10.1016/S0363-8111(97)90023-0 
Bhattacharjee, A., Berman, J. Z., \& Reed, A. (2013). Tip of the hat, wag of the finger: How moral decoupling enables consumers to admire and admonish. Journal of Consumer Research, 39(6), 1167-1184. https://doi.org/10.1086/667786

Birkner, T., \& Nölleke, D. (2016). Soccer players and their media-related behavior: a contribution on the mediatization of sports. Communication \& Sport, 4(4), 367-384. https://doi.org/10.1177/ 2167479515588719

Blaney, J. R., Lippert, L., \& Smith, J. S. (Hrsg.) (2013). Repairing the athlete's image. Studies in sports image restoration. Lanham, MD: Lexington Books.

Böcking, T. (2007). Sportskandale in der Presse [Sports scandals in the press]. Publizistik, 52(4), 502-523. https://doi. org/10.1007/s11616-007-0245-1

Brown, K.A., Dickhaus, J., \& Long, M. C. (2012). LeBron James and, the decision': an empirical examination of image repair in sports. Journal of Sports Media, 7(1), 149-175. https://doi. org/10.1353/jsm.2012.0010

Buschmann, R., \& Wulzinger, M. (2017). Football Leaks. Die schmutzigen Geschäfte im Profifußball [Football Leaks. The dirty business of professional football]. München: Deutsche Verlags-Anstalt.

Easton, D. (1953). The political system: An inquiry into the state of political science. New York: Knopf.

Festinger, L. (1957). A theory of cognitive dissonance. Stanford: Stanford University Press.

Fleischhauer, J. (2018, 21.03). Vorwurf des Steuerbetrugs. Staatsanwaltschaft verlangt fünf Jahre Haft für Xabi Alonso [Allegation of tax fraud. Prosecutor demands five years imprisonment for Xabi Alonso]. Spiegel Online. Verfügbar unter: http://www.spiegel.de/sport/ fussball/xabi-alonso-staatsanwalt- schaft-fordert-fuenf-jahre-haft-wegensteuervergehens-a-1199156.html [18.12.2018].

Fürtjes, O., \& Hagenah, J. (2011). Der Fußball und seine Entproletarisierung [Football and its deproletarisation]. KZfSS Kölner Zeitschrift für Soziologie und Sozialpsychologie, 63(2), 279-300. https:// doi.org/10.1007/s11577-011-0132-7

Gleich, U. (2001). Sportberichterstattung in den Medien: Merkmale und Funktionen. Ein Forschungsüberblick [Sports reporting in the media: features and functions. A research overview]. In G. Roters, W. Klingler, \& M. Gerhards (Hrsg.), Sport und Sportrezeption, Schriftenreihe Forum Medienrezeption, Band 5, (S. 167-182). Baden-Baden: Nomos.

Haberstroh, K., Orth, U., Hoffmann, S., \& Brunk, B. (2017). Consumer response to unethical corporate behavior: a reexamination and extension of the moral decoupling model. Journal of Business Ethics, 140(1), 161-173. https:// doi.org/10.1007/s10551-015-2661-x

Hayes, A. F. (2018). Introduction to mediation, moderation, and conditional process analysis. A regression-based approach (2nd Edition). New York: Guilford Press.

Heider, C., \& Nufer, G. (2013). Wirkungen von Testimonialwerbung mit prominenten Sporterln - Ergebnisse einer empirischen Untersuchung [Effects of testimonial advertising with prominent athletes - results of an empirical study]. Sciamus - Sport und Management, (2), 1-17.

Heil, L. U. (2016). Gesättigte Demokratie. Ein marketingorientierter Alternativbegriff zur Politikverdrossenheit [Saturated democracy. A marketing-oriented alternative concept to the disenchantment with politics]. Wiesbaden: Springer VS. 
Heinecke, S. (2014). Fit fürs Fernsehen?: die Medialisierung des Spitzensports als Kampf um Gold und Sendezeit [Fit for television?: the medialization of topclass sport as a battle for gold and broadcasting time]. Köln: Herbert von Halem.

Hummel, T. (2017, 22.06). Steuern zahlen? Wir sind doch Fußballer! [Pay taxes? We're soccer players!] Süddeutsche Zeitung [Online]. Verfügbar unter: http:// www.sueddeutsche.de/sport/fussballsteuern-zahlen-wir-sind-doch-fussballer-1.3554976 [21.09.2017].

Jarren, O., \& Donges, P. (2011). Politische Kommunikation in der Mediengesellschaft. Eine Einführung [Political communication in the media society. An introduction] (3. überarb. Aufl.). Wiesbaden: VS.

Kepplinger, H. M. (2009). Publizistische Konflikte und Skandale [Journalistic conflicts and scandals]. Wiesbaden: VS.

Koch, T., Peter, C., \& Müller, P. (2019). Das Experiment in der Kommunikations-und Medienwissenschaft. Grundlagen, Durchführung und Auswertung experimenteller Forschung [The experiment in communication and media research. Basics, implementation and analysis of experimental research]. Wiesbaden: Springer VS.

Lee, J. S., Kwak, D. H., \& Braunstein-Minkove, J. R. (2016). Coping with athlete endorsers' immoral behavior: roles of athlete identification and moral emotions on moral reasoning strategies. Journal of Sport Management, 30(2), 176-191. https://doi.org/10.1123/jsm.2015-0341

Lee, J.S., Kwak, D. H., \& Moore, D. J. (2015). Athletes' transgressions and sponsor evaluations: a focus on consumers' moral reasoning strategies. Journal of Sport Management, 29(6), 672-687. https://doi.org/10.1123/JSM.2015-0051
Liu, B. F. (2007). President Bush's major post-Katrina speeches: enhancing image repair discourse theory applied to the public sector. Public Relations Review, 33(1), 40-48. https://doi.org/10.1016/j. pubrev.2006.11.003

Meng, J., \& Pan, P. L. (2013). Revisiting image-restoration strategies: an integrated case study of three athlete sex scandals in sports news. International Journal of Sport Communication, 6(1), 87-100. https://doi.org/10.1123/ijsc.6.1.87

Meyen, M. (2014). Medialisierung des deutschen Spitzenfußballs. Eine Fallstudie zur Anpassung von sozialen Funktionssystemen an die Handlungslogik der Massenmedien [Medialization of German professional soccer. A case study concerning the adaptation of social functional systems to the logic of mass media]. Medien und Kommunikationswissenschaft, 62(3), 377-394. https:// doi.org/10.5771/1615-634x-2014-3-377

Nölleke, D., \& Blöbaum, B. (2012). Medial dabei statt mittendrin? Sportzuschauer als Medienrezipienten [Sports spectators as media recipients]. In B. Strauß (Hrsg.), Sportzuschauer (S. 162-179). Göttingen: Hogrefe.

Raney, A. A. (2008). Motives for using sport in the media: Motivational aspects of sport reception process. In $\mathrm{H}$. Schramm (Hrsg.), Die Rezeption des Sports in den Medien (S. 52-77). Köln: Herbert von Halem.

Schulz, W. (2011). Politische Kommunikation. Theoretische Ansätze und Ergebnisse empirischer Forschung [Political communication. Theoretical approaches and empirical results] (3. überarb. Aufl.). Wiesbaden: VS.

Sheldon, C. A., \& Sallot, L. M. (2008). Image repair in politics: testing effects of communication strategy and performance history in a faux pas. Journal of Public 
Relations Research, 21(1), 25-50. https:// doi.org/10.1080/10627260802520496

Staatsanwaltschaft zeigt Cristiano Ronaldo wegen Steuerhinterziehung an [Public prosecutor's office accuses Cristiano Ronaldo of tax evasion] $(2017,13.06)$. Süddeutsche Zeitung [Online]. Verfügbar unter: https://www.sueddeutsche.de/ sport/fussball-staatsanwaltschaft-zeigtcristiano-ronaldo-wegen-steuerhinterziehung-an-1.3544530 [18.12.2018].

Stiehler, H.-J. (2007). Sportrezeption zwischen Unterhaltung und Information [Sports reception between entertainment and information]. In T. Schierl (Hrsg.), Handbuch Medien, Kommunikation und Sport (S. 182-199). Schorndorf: Hofmann-Verlag.
Symmank, C., \& Hoffmann, S. (2017). Leugnung und Ablehnung von Verantwortung [Denial and rejection of responsibility]. In L. Heidbrink, C. Langbehn, \& J. Loh (Hrsg.), Handbuch Verantwortung (S. 949-972). Wiesbaden: Springer VS.

Verdacht auf Steuerhinterziehung. Staatsanwaltschaft erhebt Anklage gegen Mourinho [Suspicion of tax evasion. Public prosecutor's office files charges against Mourinho] (2017, 20.06). Spiegel Online. Verfügbar unter: http://www.spiegel. de/sport/fussball/jose-mourinho-wirdwegen-steuerhinterziehung-in-spanienangeklagt-a-1153113.html [18.12.2018]. 